Pacific Journal of Mathematics

REVERSIBLE HOMEOMORPHISMS OF THE REAL LINE 


\title{
REVERSIBLE HOMEOMORPHISMS OF THE REAL LINE
}

\author{
ARnold B. CALiCA
}

\begin{abstract}
Let $G$ be the group of germs of $C^{k}$ local homeomorphisms of the real line which fix the origin and have nonzero derivative there. In this paper the possibility of factoring an element of $G$ which is conjugate to its inverse into the product of two involutions is investigated. It is shown that it is always possible to do this in the analytic case and not always possible in the continuous case. In the intermediate cases several necessary and sufficient conditions are developed for determining whether or not such a factorization is possible. Included is a construction which allows one to determine an explicit factorization. Indication is given of the application of this material to the same problem in higher dimensions. This work is related to some material in Dynamics.
\end{abstract}

1. Introduction. If $G$ is an abstract group an element $g \in G$ is called reversible in $G$ if there exists an element $h \in G$ such that $h g h^{-1}=g^{-1}$. The product of two involutions is always reversible by an obvious argument. There arises the following question:

Question \#. If $g$ is reversible in $G$ can $g$ be factored into the product of two involutions in $G$ ?

D. C. Lewis has decided this issue in the case $G=G L(n, C)$ affirmatively (Lewis [4]).

This paper concerns itself with the investigation of this question in the case where $G$ is the group of germs of continuous or differentiable homeomorphisms of the real line.

Reversible transformations play a role in Dynamics. For further information on this connection see the references in Lewis [4].

2. Definitions and Notation. $C^{k}=\{F: F$ is a local homeomorphism of a neighborhood of 0 in $\boldsymbol{R}$ to another such neighborhood which fixes the origin and is of class $C^{k}$ on some neighborhood of 0 , $F^{\prime}(0) \neq 0$ if $k>0$, for $0 \leqq k \leqq \infty$ or analytic for $\left.k=\omega\right\} . \quad T^{k}=$ \{germs of elements of $\boldsymbol{C}^{k}$ \}

Let $\phi_{k}: \boldsymbol{C}^{k} \rightarrow \boldsymbol{T}^{k}$ be the map which assigns to each element of $\boldsymbol{C}^{k}$ its germ in $\boldsymbol{T}^{k}$. The binary operation of (local) composition of mappings in $C^{k}$ induces a group multiplication in $\boldsymbol{T}^{k} . \boldsymbol{T}^{k}$ will be viewed as a group with this structure henceforward. 
If a property is locally true for each $\alpha \in f, \alpha \in \boldsymbol{C}^{k}, f \in \boldsymbol{T}^{k}$ the property will be attributed to $f$. The identity element of any group under consideration will be called 1 .

$\boldsymbol{P}^{k}=\left\{\right.$ set of all real power series of the form $\sum_{r=1}^{k} a_{r} x^{r}, a_{1} \neq 0$, $1 \leqq k \leqq \infty\}$

$\boldsymbol{P}^{\omega}=\left\{\alpha \in \boldsymbol{P}^{\infty}: \alpha\right.$ has a nonzero radius of convergence $\}$

$\boldsymbol{P}^{0}=\{1,-1\}$.

Let $\rho_{k}: \boldsymbol{T}^{k} \rightarrow \boldsymbol{P}^{k}, k>0$ be the mapping which assigns to each element of $\boldsymbol{T}^{k}$ its Taylor expansion to degree $k$ about the origin. Put $\rho_{0}(f)= \pm 1$ according as $f$ is the germ of a locally increasing or locally decreasing element of $\boldsymbol{C}^{0}$.

REMARK 1. If $0 \leqq k \leqq \infty$ or $k=\omega, \rho_{k}$ is onto. This trivial for $k<\infty$ familiar for $k=\omega$ and true for $k=\infty$. For the last case see Borel [2].

The multiplication in $\boldsymbol{T}^{k}$ induces via the mapping $\rho_{k}$ a group multiplication in $\boldsymbol{P}^{k} . \quad \boldsymbol{P}^{k}$ will be viewed as a group with this multiplication. The elements of $\boldsymbol{P}^{k}$ are often referred to as jets in the literature. Note that composition of elements in $\boldsymbol{P}^{k}$ is not multiplication of polynomials but is substitution followed by truncation to degree $k$. Other homomorphisms which will be found useful are the mappings $\boldsymbol{T}^{k} \rightarrow \boldsymbol{T}^{m}$ and $\boldsymbol{P}^{k} \rightarrow \boldsymbol{P}^{m}, m \leqq k$ defined by the inclusion mapping of $\boldsymbol{C}^{k} \rightarrow \boldsymbol{C}^{m}$ followed by $\phi_{m}$ or $\rho_{m} \phi_{m}$.

For $F \in C^{k}$, let $F_{+}$(resp., $F$ ) be the restriction of $F$ to $R_{+}=$ $\{x \in \boldsymbol{R}: x \geqq 0\}$ (resp., to $\boldsymbol{R}_{-}=\{x \in \boldsymbol{R}: x \leqq 0\}$ ). $F_{+}$(resp., $F_{-}$) is a local homeomorphism fixing 0 of $\boldsymbol{R}_{+}$to $\boldsymbol{R}_{+}$or $\boldsymbol{R}_{+}$to $\boldsymbol{R}_{-}$(resp., $\boldsymbol{R}_{-}$to $\boldsymbol{R}_{-}$or $\boldsymbol{R}_{-}$to $\left.\boldsymbol{R}_{+}\right)$. The notation $f_{+}$and $f_{-}$will denote the corresponding notion for the germ $f . f_{+}^{-1}$ (resp., $\left.f_{-}^{-1}\right)$ will denote $\left(f_{+}\right)^{-1}\left(\operatorname{resp} .,\left(f_{-}\right)^{-1}\right)$.

3. Periodic local homeomorphisms of $\boldsymbol{R}$. Let

$\boldsymbol{T}_{+}^{k}=$ the set of elements of $\boldsymbol{T}_{-}^{k}$ which are increasing,

$\boldsymbol{P}_{+}^{k}=\rho_{k}\left(\boldsymbol{T}_{+}^{k}\right)$

$\boldsymbol{T}_{-}^{k}=$ the set of elements of $\boldsymbol{T}^{k}$ which are decreasing,

$\boldsymbol{P}_{-}^{k}=\rho_{k}\left(\boldsymbol{T}_{-}^{k}\right)$

\section{REMARK 2.}

(i) $\boldsymbol{T}_{+}^{k} \cap \boldsymbol{T}_{-}^{k}=\varnothing, \boldsymbol{T}_{+}^{k} \cup \boldsymbol{T}_{-}^{k}=\boldsymbol{T}^{k}$

(ii) $\boldsymbol{T}_{+}^{k}$ is a subgroup of $\boldsymbol{T}^{k}$ which is of index 2 and is therefore normal.

(iii) $\boldsymbol{T}_{+}^{k} \boldsymbol{T}_{-}^{k}=\boldsymbol{T}_{-}^{k} \boldsymbol{T}_{+}^{k}=\boldsymbol{T}_{-}^{k}, \boldsymbol{T}_{-}^{k} \boldsymbol{T}_{-}^{k}=\boldsymbol{T}_{+}^{k}$

The corresponding statements are true of $\boldsymbol{P}_{+}^{k}$ and $\boldsymbol{P}_{-}^{k}$.

Repeatedly used subsequently are following obvious facts:

If $f \in \boldsymbol{T}_{+}^{k}$, then $\left(f^{-1}\right)_{+}=f_{+}^{-1}$ and $\left(f^{-1}\right)_{-}=f_{-}^{-1}$. 
If $f \in \boldsymbol{T}_{-}^{k}$, then $\left(f^{-1}\right)_{+}=f_{-}^{-1}$ and $\left(f^{-1}\right)_{-}=f_{+}^{-1}$.

Proposition 1. If $g \in \boldsymbol{T}_{+}^{k}, g^{m}=1$ for some integer $m \neq 0$, then $g=1$.

CoROLlaRY. If $g \in \boldsymbol{T}^{k}$ and $g^{m}=1$ for some integer $m \neq 0$, then $g^{2}=1$.

Proof of Proposition 1. Let $G \in C^{k}$ such that $\phi_{k}(G)=g$ and $g^{m}=$ 1. $G$ is clearly monotone near 0 . Thus for small $x>0$, if $x<G(x)$, then $x<G(x)<\cdots<G^{m}(x)=x$. This is impossible. If $x>G(x)$ a similar argument applies. This constitutes a proof of this proposition.

It is to be noted that Proposition 1 follows trivially from a theorem in Bochner [1] in the case $k>0$.

REMARK 2. $f \in \boldsymbol{T}_{-}^{k}$ and $f^{2}=1$, if and only if $f_{-}=f_{+}^{-1}$.

4. Factorization of Reversible Transformations of the Real line. In this section the possibility of factoring a reversible element of $\boldsymbol{T}^{k}$ is investigated.

Df: If $f \in \boldsymbol{T}_{-}^{k}$, then $\bar{f}$ is the element of $\boldsymbol{T}^{0}$ such that,

$$
(\bar{f})_{+}=f_{+} \text {and }(\bar{f})_{-}=f_{+}^{-1} \text {. }
$$

Lemma 1. If $f \in \boldsymbol{T}_{-}^{k}$ and $\rho_{k}(f)$ is an involution in $\boldsymbol{P}^{k}$, then $\bar{f}$ is an involution in $\boldsymbol{T}^{k}$.

Proof. $\left(f^{-1}\right)_{+}=f_{-}^{-1}$ and $\left(f^{-1}\right)_{-}=f_{+}^{-1}$ since $f \in \boldsymbol{T}_{-}^{k} . \quad \rho_{k}\left(f^{2}\right)=1 \mathrm{im}-$ plies $\rho_{k}\left(f^{-1}\right)=\rho_{k}(f)$. Therefore the right derivatives of $f_{+}$at zero are the same as the left derivatives of $f_{+}^{-1}$ at zero. Therefore, $\bar{f} \in \boldsymbol{T}^{k}$ and $\bar{f}^{2}=1$ by Remark 2 .

THEOREM 1. If $f \in \boldsymbol{T}_{+}^{k}(0 \leqq k \leqq \infty$ or $k=\omega)$, then $f$ is the product of two involutions in $\boldsymbol{T}^{m},(m \leqq k)$ if and only if there exists $g \in \boldsymbol{T}_{-}^{m}$ such that $g f g^{-1}=f^{-1}$ and $\rho_{m}(g)$ is an involution in $\boldsymbol{P}^{m}$.

Proof of Theorem 1. If $f=h k, h, k \in \boldsymbol{T}^{m}$ and $h^{2}=k^{2}=1$, then by Remark 2 and Proposition 1 and its corollary one can conclude that either $h, k \in \boldsymbol{T}^{m}$ or $f=1$. If $h, k \in \boldsymbol{T}_{-}^{m}$, set $g=h$. If $f=1$ set $g$ equal to any involution in $\boldsymbol{T}_{-}^{m}$. In either case $\rho_{m}(g)$ is also an involution.

Assume now that $f^{-1}=g f g^{-1}$ where $g \in \boldsymbol{T}_{-}^{m}$ and $\rho_{m}(g)$ is an involution. If one sets $(\bar{g})_{+}=g_{+},(\bar{g})_{-}=g_{+}^{-1}$ it is then easy to verify that $g f g^{-1}=f^{-1}$ implies $\bar{g} f \bar{g}^{-1}=f^{-1}$. This means that $f=\bar{g}(\bar{g} f)$ and 
both factors are involutions.

Some of the preceding material can be utilized to demonstrate the impossibility of factoring each reversible element of $\boldsymbol{T}^{0}$ into the product of two involutions. This is embodied in the following proposition:

Proposition 3. There exists a reversible element of $\boldsymbol{T}^{0}$ which cannot be factored into the product of two involutions.

Proof of Proposition 3. Suppose there exists an element $f \in \boldsymbol{T}_{+}^{0}$ such that $f$ is reversible and $f_{-}=1$ and $f_{+} \neq 1$. It is clear that $g f g^{-1}=f^{-1}$ implies that $g \in \boldsymbol{T}_{+}^{0}$. If such an element exists it cannot be factored as the product of two involutions by Theorem 1. An element of this type is constructed below:

Let $f=\phi_{0}(F)$ where $F_{-}=x, F \in C^{0}$

$$
\text { and } F_{+}=\left\{\begin{array}{cc}
F_{0} & x \in(1 / 2,1] \\
F_{1} & x \in(1 / 4,1 / 2] \\
\vdots & \\
F_{k} & x \in\left(2^{-k-1}, 2^{-k}\right] \\
\vdots & \vdots
\end{array}\right.
$$

and

$$
F_{k+1}=\hat{F}_{k} \text { and } \hat{F}_{k}(x)=\left(1 / 2 F_{k}(2 X)\right)^{-1}
$$

and

$$
F_{0}(x)= \begin{cases}x+\frac{1}{\mathrm{M}} \exp \left[(x-1)^{-1}-(x-1 / 2)^{-1}\right] & x \in(1 / 2,1) \\ 1 & x=1\end{cases}
$$

with $M$ large enough to ensure that $F_{0}$ is one to one on its domain of definition. It can be easily verified that $F$ is continuous on $(-\infty, 1]$. Moreover $2 F(1 / 2 x)=F^{-1}(x)$. Therefore $f=\phi_{0}(F)$ is reversible and not the product of two involutions. This completes the proof of the proposition.

REMARK 3. Composition in the group $\boldsymbol{P}^{n}$ is given as follows: If

$$
\alpha=\sum_{k=1}^{n} a_{k} x^{k} \quad \text { and } \quad \beta=\sum_{k=1}^{n} b_{k} x^{k},
$$

then 


$$
\beta \alpha=\sum_{k=1}^{n} A_{k} x^{k}
$$

where

$$
\begin{gathered}
A_{k}=\sum \frac{r !}{r_{1} ! r_{2} ! \cdots r_{k} !} b_{r} a_{1}^{r_{1}} a_{2}^{r_{2}} \cdots a_{k}^{r_{k}}, \\
r_{1}+2 r_{2}+\cdots+k r_{k}=k
\end{gathered}
$$

and

$$
r=r_{1}+r_{2}+\cdots+r_{k} \text {. }
$$

Lemma 2. Suppose $\alpha \in \boldsymbol{P}^{k}, m \leqq k$

$$
\text { If } \alpha=x+a_{n} x^{m}+o\left(x^{m}\right),
$$

then

$$
\alpha^{-1}=x-a_{m} x^{m}+o\left(x^{m}\right) .
$$

$$
\text { If } \alpha=-x+a_{m} x^{m}+o\left(x^{m}\right) \text {, }
$$

then

$$
\alpha^{-1}=-x+(-1)^{m} a_{m} x^{m}+o\left(x^{m}\right) .
$$

Lemma 3. Suppose $\alpha \in \boldsymbol{P}^{k}, \alpha$ is reversible, $m \leqq k$ and $\alpha=$ $x+a_{m} x^{m}+o\left(x^{m}\right), a_{m} \neq 0$.

If $\beta \alpha \beta^{-1}=\alpha^{-1}$ and $\beta=\sum_{s=1}^{k} b_{s} x^{s}$, then $b_{1}=-1$. Moreover, $m$ must be even.

Lemma 4. If $\alpha \in \boldsymbol{P}^{2}$ and $\alpha=-x+a_{2} x^{2}$, then $\alpha$ is an involution.

The verification of Lemmas $2-4$ is straightforward and will therefore be omitted.

$D f:$ If $f \in \boldsymbol{T}^{k}, k>0, m \leqq k$, let

$$
f^{(m)}(0)=\left.\frac{d^{m} F(x)}{d x^{m}}\right|_{x=0} \quad \text { for any } F \in C^{k}
$$

such that $\phi_{k}(F)=f$.

THEOREM 2. If $f \in \boldsymbol{T}_{+}^{k}, k \geqq 2, f$ reversible and $\rho_{k}(f) \neq 1$ then $f$ can be factored as the product of two involutions in $\boldsymbol{T}^{2}$.

Proof of Theorem. Let $f^{-1}=g f g^{-1}$; Lemma 3 and Lemma 4 show 
that $\rho_{2}(g)$ is an involution; now Theorem 1 applies to the image of $f$ in $T^{2}$.

CoRollary. If $f \in \boldsymbol{T}_{+}^{\omega}$ and $f$ reversible in $\boldsymbol{T}^{\omega}$, then $f$ can be factored as the product of two involutions in $\boldsymbol{T}^{2}$.

This follows from Theorem 2 and the observation that if $f \in \boldsymbol{T}_{+}^{\omega}$ and $f^{(m)}(0)=0$ for $m>0$, then $f=1$.

The question of factorization of reversible elements of $\boldsymbol{T}_{-}^{\omega}$ is settled positively in the following material.

Lemma 5. If $g, h \in \boldsymbol{T}_{+}^{\omega}$ and $g^{2}=h^{2}$, then $g=h$.

Proof of Lemma 5. If $g-h$ has zeros in every neighborhood of 0 , then $g=h$ since $g$ and $h$ are analytic. Assume therefore that there exists a neighborhood $(0, \varepsilon], \varepsilon>0$, such that $g(x)>h(x)$ and $g(x), h(x)$ are monotone in $(0, \varepsilon]$. Choose $x_{0}>0$ sufficiently small such that $x_{0}$, $g\left(x_{0}\right), h\left(x_{0}\right), g^{2}\left(x_{0}\right) \in(0, \varepsilon]$. Then $g\left(x_{0}\right)>h\left(x_{0}\right)$ and $g^{2}\left(x_{0}\right)>g h\left(x_{0}\right)>h^{2}\left(x_{0}\right)$. This contradicts $g^{2}=h^{2}$.

Theorem 3. If $f \in \boldsymbol{T}_{-}^{\omega}$ and $f$ is reversible in $\boldsymbol{T}^{\omega}$, then $f$ is an involution in $\boldsymbol{T}^{\omega}$ (hence $1 \cdot f$ is the product of two involutions in $\left.\boldsymbol{T}^{\omega}\right)$.

Proof of Theorem 3. Suppose $f^{-1}=g f g^{-1}$. The proof of this theorem is divided into two cases according as $g \in \boldsymbol{T}_{+}^{\omega}$ or $g \in \boldsymbol{T}_{-}^{\omega}$.

Case I. $g \in \boldsymbol{T}_{+}^{\omega}$.

Moreover, $g f g^{-1}=f^{-1}$ iff $g_{-} f_{+} g_{+}^{-1}=f_{-}^{-1}$ and $g_{+} f_{-} g_{-}^{-1}=f_{\mp}^{-}$iff $f_{-}=$ $g_{+} f_{+}^{-1} g_{-}^{-1}$ and $f_{-}=g_{+}^{-1} f_{+}^{-1} g_{-}$

$$
\text { iff }\left\{\begin{array}{l}
f_{-}=g_{+} f_{+}^{-1} g_{-}^{-1} \\
g_{+}^{2}=f_{+}^{-1} g_{-}^{2} f_{+}=\left(f_{+}^{-1} g_{-} f_{+}\right)^{2}
\end{array}\right.
$$

Since $g_{+}$is locally increasing and analytic the foregoing implies that

$$
g_{+}=f_{+}^{-1} g_{-} f_{+}
$$

using Lemma 5.

Therefore

$$
\begin{aligned}
& f_{-}=g_{+} f_{-}^{-1} g_{-}^{-1} \quad \text { by }(\#) \text {. } \\
& f_{-}=\left(f_{+}^{-1} g_{-} f_{+}\right) f_{+}^{-1} g_{-}^{-1} \quad \text { by }\left(^{*}\right) \text {. }
\end{aligned}
$$

Therefore $f_{-}=f_{+}^{-1}$ so $f$ is an involution. 
Case II. Suppose $g \in \boldsymbol{T}_{\stackrel{\omega}{\omega}}$.

Therefore, $g f g^{-1}=f^{-1}$ is equivalent to $g_{+} f_{-} g_{-}^{-1}=f_{-}^{-1}$ and $g_{-} f_{+} g_{+}^{-1}=$ $f_{+}^{-1}$. The foregoing is equivalent to $g_{-}=f_{-} g_{+} f_{-}$and $g_{-}=f_{+}^{-1} g_{+} f_{+}^{-1}$. This means $g_{-} g_{+}=\left(f_{-} g_{+}\right)^{2}$ and $g_{-} g_{+}=\left(f_{+}^{-1} g_{+}\right)^{2}$. Therefore, $\left(f_{-} g_{+}\right)^{2}=\left(f_{+}^{-1} g_{+}\right)^{2}$. Lemma 5 implies $f_{-} g_{+}=f_{+}^{-1} g_{+}$. This means that $f_{-}=f_{+}^{-1}$. The last statement and Remark 2 implies that $f$ is an involution. This concludes the proof of this theorem.

REMARK. It is known that Lemma 5 is false in $\boldsymbol{T}^{0}$. It is not known if Lemma 5 is true in $\boldsymbol{T}^{k} 1 \leqq k \leqq \infty$. The truth of Lemma 5 for $\boldsymbol{T}^{k}$ would permit one to state the analogue of Theorem 6 for $\boldsymbol{T}^{k}$. If Lemma 5 is false for $\boldsymbol{T}^{k}$ one could conclude that reversible elements of $\boldsymbol{T}^{k}$ could not be factored into the product of two involutions.

There is a square root lemma, weaker than Lemma 5, which will be proved which provides an additional criterion for the factorability of a reversible element of $\boldsymbol{T}^{k}$ into the product of two involutions.

Lemma 6. If $f, g \in \boldsymbol{T}_{+}^{k}, f^{\prime}(0)=a \neq 1, k \geqq 2, f^{2}=g^{2}$, then $f=g$.

Proof of Lemma 6. If $f^{2}=g^{2}=l$, then $l^{\prime}(0)=a^{2}$. By Sternberg [5] Theorem 2 there exists $h \in T_{+}^{k-1}$ such that $h l h^{-1}=\phi_{k-1}\left(a^{2} x\right)$. It is now shown that the only differentiable square root of $\phi_{k-1}\left(a^{2} x\right)$ in $\boldsymbol{T}_{+}^{k-1}$ is $\phi_{k-1}(a x)$. Suppose there is another square root of $\phi_{k-1}\left(a^{2} x\right)$ given by $\phi_{k-1}(K(x))$. Consider some set $(0, \eta)$ on which $K^{2}(x)=a^{2} x,(\eta>0)$. It is clear that $K(x)>a x$ or $K(x)<a x$ cannot hold in $(0, \eta)$. Therefore there is some point $x_{0} \in(0, \eta)$ where $a x=K(x)$. Assume further that $a<1$. If this is not the case one can apply the argument to $l^{-1}$.

In the argument following it will be convenient to utilize the notation $\left(F^{m}\left(y_{0}\right)\right)^{\prime}$ for $d /\left.d x F^{m}(x)\right|_{x=y_{0}}$.

Let $x_{k}=K^{k}\left(x_{0}\right)=a^{k} x_{0}$. Since $a<1 \lim _{k \rightarrow \infty} x_{k}=0$ monotonically. $K^{\prime}(x) \neq a x$ at some point in $\left(x_{1}, x_{0}\right)$; otherwise $K(x)=a x$ on $(0, \eta)$ and there is nothing to prove. Assume, therefore, that there exists $y_{0} \in\left(x_{1}, x_{0}\right)$ such that $K^{\prime}\left(y_{0}\right)=\beta \neq a$. Let $Z_{k}=K^{2 k-1}\left(y_{0}\right)$. Since $x_{2 k}<Z_{k}<x_{2 k-1}$ one observes that $\lim _{k \rightarrow \infty} Z_{k}=0$. Since $K^{2}(x)=a^{2} x$, one observes that $K^{\prime}(0)=a$ by the chain rule and the fact that $K^{\prime}(0)>0$. This implies $\lim _{k \rightarrow \infty} K^{\prime}\left(Z_{k}\right)=a$.

But,

$$
\begin{aligned}
K^{\prime}\left(Z_{k}\right) & =K^{\prime} K^{2 k-1}\left(y_{0}\right)=\frac{\left(K^{2 k}\left(y_{0}\right)\right)^{\prime}}{\left(K^{2 k-1}\left(y_{0}\right)\right)^{\prime}} \\
& =\frac{\left(K^{2 k}\left(y_{0}\right)\right)^{\prime}}{\left.\left(K^{2 k-2}\left(y_{0}\right)\right)^{\prime} K\left(y_{0}\right)\right)^{\prime}}
\end{aligned}
$$


Therefore,

$$
K^{\prime}\left(Z_{k}\right)=\frac{a^{2 k}}{a^{2 k-2} \beta}=\frac{a^{2}}{\beta} \neq a .
$$

Therefore $\lim _{k \rightarrow \infty} K^{\prime}\left(Z_{k}\right) \neq K^{\prime}(0)$ which contradicts the continuity of $K^{\prime}(x)$. This means that $\phi_{k-1}(a x)$ is the only square root of $\phi_{k-1}\left(a^{2} x\right)$ in $\boldsymbol{T}_{+}^{k-1}(R, 0)$.

Since $h f h^{-1}$ and $h g h^{-1}$ are differentiable square roots of $\phi_{k-1}\left(a^{2} x\right)$, $h f h^{-1}=h g h^{-1}$ which implies $f=g$. This concludes the proof.

The foregoing leads to the following theorem.

THeorem 4. If $f \in \boldsymbol{T}_{-}^{k}, k \geqq 2, f$ reversible in $\boldsymbol{T}_{-}^{k}$ and $f^{-1}=g f g^{-1}$ $g \in \boldsymbol{T}_{+}^{k}$ and $g^{\prime}(0) \neq 1$, then $f$ is an involution.

Proof of Theorem 4. The proof utilizes the construction in the proof of Theorem 3 and will therefore be omitted.

5. Conclusions. In this paper Question $(\sharp)$ of the introduction is shown to have answer "no" for continuous homeomorphisms and nearly "yes" for analytic homeomorphisms. For $\boldsymbol{C}^{k}$ homeomorphisms, $1 \leqq k \leqq \infty$, the reply to the Question is shown to depend on the existence of unique square roots in a particular group and the nonexistence of an element $f \in \boldsymbol{T}_{+}^{k}$ such that $\rho_{k}(f)=1$ and $f$ is reversible in $\boldsymbol{T}_{+}^{k}$. It is not known whether $g f g^{-1}=f^{-1}$ in $\boldsymbol{T}^{\omega}, f \in \boldsymbol{T}_{+}^{\omega}, f \neq 1$ implies that $\bar{g}$ is more than twice differentiable. One conjectures that this is not the case since it may be shown that $\alpha \beta \alpha^{-1}=\beta^{-1}$ in $P^{3}, \beta \neq 1$ and $\beta=x+b_{3} x^{3}$, does not ensure that $\alpha^{2}=1$.

One may generalize some of this material to higher dimensions. The techniques of this paper are applicable to some germs of transformations of $\boldsymbol{R}^{n}$ which fix a sufficiently nice $n-1$ manifold containing the origin. This and related questions will be treated in a subsequent paper.

\section{REFERENCES}

1. S. Bochner, Compact groups of differentiable transformations, Annals of Math., 47 (1946), 639-653.

2. Emile Borel, Sur quelques points de la theorie des fonctions, Paris, Gauthier Villars et Fils, 1894.

3. A. B. Calica, Reversible Homeomorphisms of the Real Line, Thesis, Johns Hopkins University, Baltimore, 1970.

4. D. C. Lewis, Jr., Reversible Transformations, Pacific J. Math. Vol. II, No. 3, (1961), 1077-1087.

5. S. Sternberg, Local $C^{n}$ Transformations of the Real Line, Duke Math. J. 24 (1957), 97-102. 
Received May 22, 1970. This material is part of the author's dissertation written under the supervision of Professor D. C. Lewis, Jr. of Johns Hopkins University. This work was supported in part by a grant from the Naval Academy Research Council. The referee and Professor Samelson corrected a number of obscurities in the original exposition. In addition the proofs of Proposition 1 and Theorem 2 are elegant alternatives to the original, provided by Professor Samelson.

UNIVERSITY OF HAWAII 



\section{PACIFIC JOURNAL OF MATHEMATICS}

\section{EDITORS}

H. SAMELSON

Stanford University

Stanford, California 94305

C. R. HobBY

University of Washington

Seattle, Washington 98105
J. DugundjI

Department of Mathematics

University of Southern California

Los Angeles, California 90007

RICHARD ARENS

University of California

Los Angeles, California 90024

\section{ASSOCIATE EDITORS}

E. F. BECKENBACH

B. H. NeUMaNN

F. WOLF

K. YoshidA

\section{SUPPORTING INSTITUTIONS}

UNIVERSITY OF BRITISH COLUMBIA

CALIFORNIA INSTITUTE OF TECHNOLOGY

UNIVERSITY OF CALIFORNIA

MONTANA STATE UNIVERSITY

UNIVERSITY OF NEVADA

NEW MEXICO STATE UNIVERSITY

OREGON STATE UNIVERSITY

UNIVERSITY OF OREGON

OSAKA UNIVERSITY
UNIVERSITY OF SOUTHERN CALIFORNIA STANFORD UNIVERSITY

UNIVERSITY OF TOKYO

UNIVERSITY OF UTAH

WASHINGTON STATE UNIVERSITY UNIVERSITY OF WASHINGTON

$\stackrel{*}{*} \stackrel{*}{*} \stackrel{*}{*}$ AMERICAN MATHEMATICAL SOCIETY
NAVAL WEAPONS CENTER 


\section{Pacific Journal of Mathematics}

Vol. 39 , No. 1

May, 1971

Charles A. Akemann, A Gelfand representation theory for $C^{*}$-algebras ....

Sorrell Berman, Spectral theory for a first-order symmetric system of

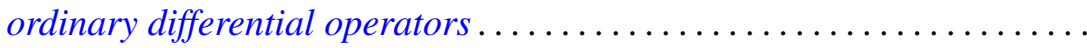

Robert L. Bernhardt, III, On splitting in hereditary torsion theories ........

J. L. Brenner, Geršgorin theorems, regularity theorems, and bounds for determinants of partitioned matrices. II. Some determinantal identities ..........................................

Robert Morgan Brooks, On representing $F^{*}$-algebras .............. 51

Lawrence Gerald Brown, Extensions of topological groups........... 71

Arnold Barry Calica, Reversible homeomorphisms of the real line ........ 79

J. T. Chambers and Shinnosuke Oharu, Semi-groups of local Lipschitzians in

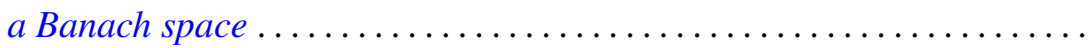

Thomas J. Cheatham, Finite dimensional torsion free rings .............

Byron C. Drachman and David Paul Kraines, A duality between

transpotence elements and Massey products ...................

Richard D. Duncan, Integral representation of excessive functions of a

Markov process ......................................

George A. Elliott, An extension of some results of Takesaki in the reduction

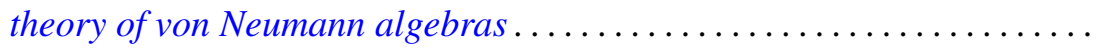

Peter C. Fishburn and Joel Spencer, Directed graphs as unions of partial

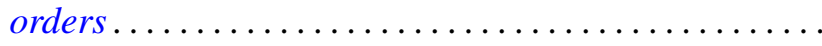

Howard Edwin Gorman, Zero divisors in differential rings ...

Maurice Heins, A note on the Löwner differential equations...

Louis Melvin Herman, Semi-orthogonality in Rickart rings. .

David Jacobson and Kenneth S. Williams, On the solution of linear G.C.D.

equations

Michael Joseph Kallaher, On rank 3 projective planes ... . .

Donald Paul Minassian, On solvable $O^{*}$-groups ...........

Nils Øvrelid, Generators of the maximal ideals of $A(\bar{D})$

Mohan S. Putcha and Julian Weissglass, A semilattice decomposition into

semigroups having at most one idempotent ............

Robert Raphael, Rings of quotients and $\pi$-regularity ....

J. A. Siddiqi, Infinite matrices summing every almost periodic sequence. .

Raymond Earl Smithson, Uniform convergence for multifunctions ...

Thomas Paul Whaley, Mulitplicity type and congruence relations in

universal algebras...

Roger Allen Wiegand, Globalization theorems for locally finitely generated modules... 